\title{
DESENVOLVIMENTO DE UM MÉTODO REFLECTOMÉTRICO AMBIENTALMENTE MAIS AMIGÁVEL PARA A DETERMINAÇÃO DE METOCLOPRAMIDA EM FORMULAÇÕES FARMACÊUTICAS
}

\author{
Keity Margareth Doretto, Mara Andréia Gotardo, Helena Redigolo Pezza e Leonardo Pezza* \\ Instituto de Química do Campus de Araraquara, Universidade Estadual Paulista Júlio de Mesquita Filho, CP 355, 14801-970 \\ Araraquara - SP, Brasil
}

Recebido em 4/3/09; aceito em 29/7/09; publicado na web em 21/1/10

\begin{abstract}
DEVELOPMENT OF AN ENVIRONMENTALLY MORE BENIGN REFLECTOMETRIC METHOD FOR THE DETERMINATION OF METOCLOPRAMIDE IN PHARMACEUTICAL FORMULATIONS. A simple and more environmentally friendly method by combined spot test-diffuse reflectance spectroscopy for determining metoclopramide in pharmaceutical formulations is described. The method is based on the reaction between metoclopramide and $p$-dimethylaminocinnamaldehyde, in the presence of $\mathrm{HCl}$, producing a colored compound $\left(\lambda_{\text {max }}=580 \mathrm{~nm}\right)$ on the filter paper. The linear range was from $5.65 \times 10^{-4}-6.21 \times 10^{-3}$ $\mathrm{mol} \mathrm{L}^{-1}(\mathrm{r}=0.999)$. The limit of detection was $1.27 \times 10^{-4} \mathrm{~mol} \mathrm{~L}^{-1}$. The proposed reflectometric method was applied successfully to the determination of metoclopramide in pharmaceuticals and it was favorably compared with the Brazilian or British Pharmacopoeia methods at $95 \%$ confidence level.
\end{abstract}

Keywords: diffuse reflectance spectroscopy; metoclopramide; spot test.

\section{INTRODUÇÃO}

A metoclopramida [4-amino-5-cloro- $N$-(2-dietil-aminoetil)2-metoxibenzamida] é uma benzamida substituída que estimula a motilidade do trato gastrintestinal superior (agente procinético) sem afetar a secreção ácida. Este fármaco aumenta o peristaltismo causando um esvaziamento gástrico acelerado. Além disso, a metoclopramida atua como antagonista da dopamina, apresentando efeito antiemético significativo, sendo indicada no tratamento de náuseas e vômitos, particularmente em associação com a quimioterapia do câncer. Outras indicações da metoclopramida incluem refluxo esofágico e estase gástrica. ${ }^{1}$

Vários métodos têm sido descritos na literatura para quantificação de metoclopramida em formulações farmacêuticas, incluindo espectrofotometria na região visível, ${ }^{2-10}$ fluorimetria, ${ }^{11}$ HPLC com detecção UV, ${ }^{12-14}$ potenciometria, ${ }^{15-18}$ espectroscopia de ressonância magnética nuclear ${ }^{19} \mathrm{e}$ voltametria. ${ }^{20} \mathrm{~A}$ Farmacopeia Brasileira ${ }^{21}$ recomenda a espectrofotometria com detecção UV para a determinação de metoclopramida em comprimidos e ampolas. O mesmo método é proposto pela Farmacopeia Britânica, ${ }^{22}$ porém acrescentando uma etapa prévia de extração com clorofórmio para eliminar a interferência de excipientes comumente presentes nas formulações. A Farmacopeia Americana ${ }^{23}$ recomenda a determinação por HPLC.

Atualmente, no desenvolvimento de métodos analíticos a quantidade, toxicidade dos reagentes ou solventes utilizados, bem como os resíduos gerados, são tão importantes quanto qualquer outra característica analítica. A maioria dos métodos analíticos descritos para a determinação de metoclopramida requer procedimentos laboriosos e morosos, seja no preparo da amostra ou no decorrer da análise, além de alguns utilizarem elevadas quantidades de solventes, gerando assim grande quantidade de resíduos, o que os enquadra fora dos padrões propostos pela Química Verde. ${ }^{24}$ Portanto, o desenvolvimento de métodos para análise de metoclopramida que sejam menos nocivos ao ambiente e que se enquadrem nos princípios preconizados pela Química Verde é uma necessidade atual e imprescindível.

Estudos descritos na literatura revelam que o uso apropriado da espectroscopia de reflectância difusa proporciona a obtenção de

*e-mail: pezza@iq.unesp.br resultados confiáveis, evidenciando a potencialidade dessa técnica para propósitos quantitativos..$^{25-32} \mathrm{Na}$ análise por reflectância, a densidade óptica para medidas de reflectância é $\mathrm{A}_{R}=-\log \mathrm{T}_{\mathrm{R}}$, análoga à absorbância, onde $\mathrm{T}_{\mathrm{R}}=\mathrm{I} / \mathrm{I}_{0}$, sendo $\mathrm{I}_{0}$ a intensidade da energia radiante incidente e I a intensidade da energia radiante refletida pelo meio. ${ }^{25}$ A combinação spot test - espectroscopia de reflectância difusa oferece vantagens sobre outros métodos, tais como simplicidade e baixo consumo de reagentes, enquadrando-a como uma metodologia ambientalmente mais amigável (benigna), pois a quantidade de resíduo gerada é muito pequena. Além disso, as medidas de reflectância podem ser realizadas in loco utilizando um equipamento portátil, o que confere uma maior versatilidade à técnica.

No entanto, relatos do uso desta técnica para análises de medicamentos ainda são incipientes. Recentemente, a espectroscopia de reflectância difusa utilizando spot test sobre papel de filtro foi empregada para a determinação de alguns fármacos em formulações farmacêuticas. ${ }^{26-28,33-35}$

Até o presente momento, não existe método descrito na literatura científica indexada para a quantificação de metoclopramida em formulações farmacêuticas por espectroscopia de reflectância difusa combinada com spot test. Desta forma, o propósito deste trabalho foi desenvolver um método analítico mais limpo, empregando esta combinação para análise de formulações farmacêuticas (comprimidos e injetáveis) contendo metoclopramida. O método baseia-se em medidas de reflectância difusa do composto violeta produzido pela reação entre metoclopramida e $p$-dimetilaminocinamaldeído ( $p$-DACA) em meio ácido, usando papel de filtro como suporte sólido. Neste estudo, as condições para a reação de spot test foram otimizadas utilizando planejamento experimental. O método desenvolvido foi aplicado com sucesso na análise de metoclopramida em formulações farmacêuticas.

\section{PARTE EXPERIMENTAL}

\section{Equipamentos}

Micropipetas Eppendorf (10 a $100 \mu \mathrm{L})$ e Brand (100 a $1000 \mu \mathrm{L})$ foram utilizadas para medidas de pequenos volumes.

Acessório de reflectância (ISP-REF, Ocean Optics, Dunedin, USA) acoplado a um espectrofotômetro portátil USB4000 (Ocean 
Optics, Dunedin, USA) foi utilizado para medidas de reflectância difusa. Para as medidas de absorbância o mesmo espectrofotômetro foi utilizado munido de cubetas de quartzo com $1 \mathrm{~cm}$ de caminho óptico.

\section{Reagentes e soluções}

Papel de filtro qualitativo Whatman foi usado como suporte sólido. Os excipientes utilizados nos estudos de interferências foram de grau farmacêutico. Foi utilizado metanol grau HPLC (Mallinckrodt, Xalostoc, México). p-dimetilaminocinamaldeído (p-DACA) (p.a., Riedel-de haën, Alemanha) foi usado para preparar a solução de p-DACA 0,8\% (m/v) em metanol. Esta solução é estável por uma semana quando mantida sob refrigeração. Soluções de trabalho de ácido clorídrico foram preparadas por diluição adequada do ácido clorídrico concentrado (37\%, Mallinckrodt, Xalostoc, México) em metanol. Solução estoque de metoclopramida (Purifarma, São Paulo, Brasil), grau de pureza $>99,79 \%$, foi preparada diariamente na concentração de $2,82 \times 10^{-2} \mathrm{~mol} \mathrm{~L}^{-1} \mathrm{em}$ metanol. Soluções padrão foram obtidas a partir de diluições da solução estoque de metoclopramida e usadas na construção da curva analítica $\left(5,65 \times 10^{-4}-6,21 \times 10^{-3} \mathrm{~mol} \mathrm{~L}^{-1}\right)$.

\section{Procedimento proposto}

As condições ótimas para a execução do procedimento foram obtidas utilizando planejamento fatorial de experimentos. Para a realização do spot test, as soluções foram adicionadas sobre o papel de filtro. Inicialmente, $10 \mu \mathrm{L}$ da solução do fármaco foram adicionados, seguidos da adição de $10 \mu \mathrm{L}$ da solução de ácido clorídrico $4,0 \%$ (v/v) e, por último, $15 \mu \mathrm{L}$ da solução de $p$-DACA $0,8 \%(\mathrm{~m} / \mathrm{v})$. As soluções foram adicionadas no centro do papel de filtro utilizando uma micropipeta adaptada na parte superior de um suporte, conforme descrito por Tubino et al. ${ }^{25}$ Todas as operações acima citadas foram realizadas em capela de boa exaustão. As medidas de reflectância difusa foram efetuadas em $580 \mathrm{~nm}$. Um branco foi preparado de maneira similar, substituindo-se a adição do fármaco por metanol.

\section{Estudo da estabilidade óptica}

Com o objetivo de avaliar a estabilidade óptica do produto colorido da reação de spot test no papel de filtro, foi realizado um acompanhamento cinético da medida de reflectância em $580 \mathrm{~nm}$ a cada 2 min até completar $1 \mathrm{~h}$.

\section{Estudos de interferentes}

O efeito dos excipientes mais comumente utilizados nas formulações farmacêuticas disponíveis no mercado (comprimidos e injetáveis) foi cuidadosamente avaliado. Os excipientes estudados foram estearato de magnésio, amido, lactose, croscarmelose sódica, polivinilpirrolidona, manitol e talco. Para este estudo, soluções contendo metoclopramida $(1,0 \mathrm{mg} / \mathrm{mL})$ e cada um dos excipientes, separadamente, em concentração igual e 10 vezes maior que a concentração de metoclopramida, foram submetidas à agitação com metanol em um agitador magnético por $10 \mathrm{~min}$. Estas misturas foram transferidas para balões volumétricos de $10,00 \mathrm{~mL}$ e o volume completado com o mesmo solvente. Em seguida, foi realizada uma filtração para separar os interferentes pouco solúveis. As soluções foram analisadas conforme descrito no procedimento proposto. Um branco contendo apenas metanol e demais reagentes foi usado como referência.

\section{Preparação e análise das amostras}

Cinco formulações farmacêuticas de diferentes marcas comerciais (três marcas de comprimidos e duas de injetáveis) foram analisadas. Os produtos foram obtidos em farmácias locais e analisados dentro de seus prazos de validade. Os comprimidos continham $10 \mathrm{mg}$ de metoclopramida por comprimido e os injetáveis $10 \mathrm{mg}$ de metoclopramida em $2 \mathrm{~mL}$ de solução, conforme declarado no rótulo pelo fabricante do produto.

Comprimidos: 20 comprimidos, do mesmo lote, de cada amostra comercial analisada foram pesados em balança analítica e triturados em gral de ágata até a obtenção de um pó fino. O valor médio da massa de um comprimido foi referente à medição de 20 comprimidos, apresentando desvio padrão relativo menor que $5 \%$, conforme proposto pela Farmacopeia Brasileira. ${ }^{21}$ Uma porção do pó equivalente à quantidade de metoclopramida presente em um comprimido (10 mg) foi pesada e submetida à agitação com cerca de $5 \mathrm{~mL}$ de metanol em um agitador magnético por $5 \mathrm{~min}$. Em seguida, a solução foi transferida para um balão volumétrico de $10,00 \mathrm{~mL}$ e o volume completado com o mesmo solvente. Esta solução foi filtrada para eliminar os excipientes insolúveis e, deste filtrado, uma alíquota de $10 \mu \mathrm{L}$ foi retirada para a reação com $p$-DACA, conforme descrito em Procedimento proposto.

Injetáveis: os conteúdos de 12 ampolas, do mesmo lote, de cada marca comercial analisada foram reunidos em um frasco e homogeneizados. Alíquota de 1,00 mL foi transferida para um balão volumétrico de 5,00 mL e o volume foi completado com metanol. Após homogeneização, uma alíquota de $10 \mu \mathrm{L}$ foi retirada para a reação com $p$-DACA, conforme descrito em Procedimento proposto.

\section{Adição de padrão}

A adição de padrão foi realizada com o objetivo de avaliar possíveis interferências provenientes da matriz nas análises. Para cada tipo de amostra (comprimido ou solução injetável) foi realizado um procedimento, conforme descrito a seguir.

Comprimidos: às amostras contendo massa equivalente a 6 mg $\left(1,69 \times 10^{-3} \mathrm{~mol} \mathrm{~L}^{-1}\right)$ de metoclopramida foram adicionados 3; 6; 9 e $12 \mathrm{mg}$ de metoclopramida p.a., correspondendo a 50, 100, 150 e $200 \%$, respectivamente, da massa de metoclopramida presente na amostra. As quantidades de metoclopramida padrão adicionadas foram estabelecidas de forma a se obter soluções com concentrações finais que abrangessem a faixa de linearidade da curva analítica. ${ }^{36}$ Ao béquer contendo amostra e metoclopramida p.a. foi adicionado metanol, seguindo o procedimento descrito em Preparação da amostra.

Injetáveis: às amostras contendo volume equivalente a $0,6 \mathrm{~mL}$ $\left(1,69 \times 10^{-3} \mathrm{~mol} \mathrm{~L}^{-1}\right)$ de metoclopramida foram adicionados 250 ; 500; 750 e $1000 \mu \mathrm{L}$ de metoclopramida padrão, correspondendo a $50,100,150$ e $200 \%$, respectivamente, da massa de metoclopramida presente na amostra. Ao béquer contendo amostra e metoclopramida p.a. foi adicionado metanol, seguindo o procedimento descrito em Preparação da amostra.

\section{Método oficial}

Os resultados obtidos pelo método proposto foram comparados estatisticamente com aqueles obtidos por um método farmacopeico oficial, aplicando-se o teste t e o teste $\mathrm{F}$ em um nível de confiança de $95 \%$. As amostras sólidas (comprimidos) foram analisadas por espectrofotometria de absorção no ultravioleta de acordo com a Farmacopeia Brasileira. ${ }^{21}$ As amostras líquidas (injetáveis) foram analisadas de acordo com a Farmacopeia Britânica, ${ }^{22}$ que também preconiza a espectrofotometria de absorção no ultravioleta para a determinação quantitativa da metoclopramida, porém incluindo uma etapa de extração com clorofórmio. 


\section{RESULTADOS E DISCUSSÃO}

O p-DACA tem apresentado um amplo uso como reagente cromogênico em análises espectrofotométricas de aminas aromáticas primárias e secundárias. ${ }^{37}$ Uma provável reação entre aminas secundárias aromáticas e $p$-DACA envolve a condensação do grupo amino secundário protonado com o grupo carbonila do reagente, formando um sal imínio ou base de Schiff. ${ }^{9,38,39}$ A maioria das reações envolvidas nos métodos descritos na literatura ocorre em meio ácido e necessita de aquecimento para a produção de compostos coloridos (variando de laranja a vermelho ou rosa).

No presente trabalho, observou-se que a metoclopramida reage com $p$-DACA, em meio ácido, produzindo a formação imediata de um produto de cor violeta intensa sobre a superfície do papel de filtro, sem a necessidade de aquecimento. A Figura 1 apresenta o espectro de reflectância com valor máximo de $\mathrm{A}_{\mathrm{R}}$ situado em $580 \mathrm{~nm}$.

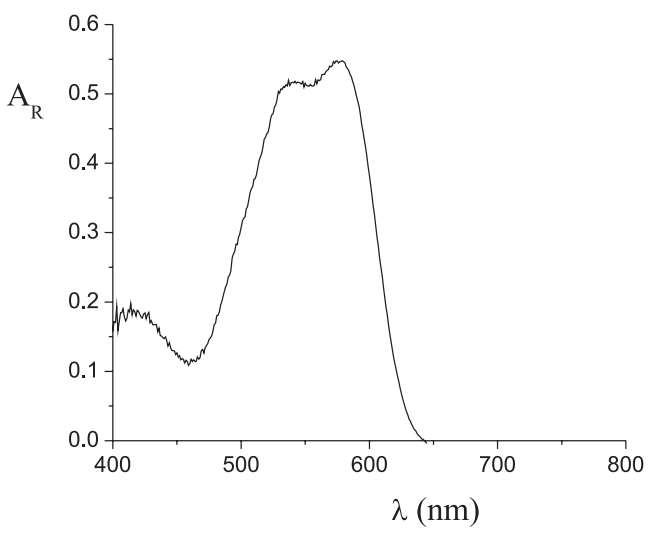

Figura 1. Espectro de reflectância do produto da reação entre metoclopramida e p-DACA, em meio ácido. [metoclopramida] $=1,69 \times 10^{-3} \mathrm{~mol} \mathrm{~L}^{-1}$

De acordo com Wendlant e Hecht, ${ }^{40}$ a cor do spot test deve ser uniforme sobre a superfície a fim de assegurar medidas de reflectância repetitíveis. Neste sentido, foram levados em consideração detalhes importantes previamente descritos ${ }^{27}$ para a reação de spot test, tais como ordem e velocidade de adição do reagente, qualidade do papel de filtro e volume das soluções adicionadas. Estes detalhes foram importantes para a uniformidade da cor do spot test, levando a uma boa precisão das medidas de reflectância.

\section{Planejamento fatorial de experimentos}

Estudos foram realizados para estabelecer as condições mais favoráveis para a reação entre metoclopramida e $p$-DACA, na presença de ácido clorídrico, sobre papel de filtro, tendo em vista alcançar a intensidade máxima da cor do produto em $580 \mathrm{~nm}$. Inicialmente, foi realizado um planejamento fatorial completo, o qual permitiu estudar simultaneamente os efeitos das principais variáveis: concentração de ácido clorídrico, concentração de $p$ DACA e a ordem de adição (O.A.) do ácido e do reagente no spot test, os quais poderiam influenciar na medida de reflectância $\left(A_{R}\right)$. O planejamento fatorial completo foi realizado em dois níveis: baixo, codificado como (-1) e alto, como $(+1) \cdot{ }^{41}$ As concentrações de $\mathrm{HCl}$ $(\%, \mathrm{v} / \mathrm{v})$ estudadas foram 4,0 (-1) e 8,0 (+1), as concentrações de $p$-DACA $(\%, \mathrm{~m} / \mathrm{v})$ foram $0,4(-1)$ e $0,8(+1)$ e as ordens de adição estudadas foram fármaco, ácido e reagente (-1) e fármaco, reagente e ácido (+1). A Tabela 1 apresenta a matriz do planejamento fatorial completo e os valores de $A_{R}$ obtidos. Os experimentos foram realizados em duplicata e seus resultados analisados pelo programa Statistica versão 6.0. Todos os experimentos foram realizados com
Tabela 1. Matriz e resultados obtidos no planejamento fatorial completo

\begin{tabular}{|c|c|c|c|c|c|}
\hline \multirow{3}{*}{$\begin{array}{c}\text { Experimento }^{\mathrm{a}} \\
1\end{array}$} & \multicolumn{3}{|c|}{ Fatores } & \multirow{2}{*}{\multicolumn{2}{|c|}{$\begin{array}{c}\text { Resposta } \\
\mathrm{A}_{\mathrm{R}}(580 \mathrm{~nm})\end{array}$}} \\
\hline & \multirow{2}{*}{$\frac{[\mathrm{HCl}]^{\mathrm{b}}}{-1}$} & \multirow{2}{*}{$\frac{[p-\mathrm{DACA}]^{\mathrm{c}}}{-1}$} & \multirow{2}{*}{$\frac{\text { O.A. }{ }^{\mathrm{d}}}{-1}$} & & \\
\hline & & & & 0,607 & 0,636 \\
\hline 2 & +1 & -1 & -1 & 0,599 & 0,613 \\
\hline 3 & -1 & +1 & -1 & 0,674 & 0,716 \\
\hline 4 & +1 & +1 & -1 & 0,669 & 0,655 \\
\hline 5 & -1 & -1 & +1 & 0,576 & 0,546 \\
\hline 6 & +1 & -1 & +1 & 0,286 & 0,334 \\
\hline 7 & -1 & +1 & +1 & 0,608 & 0,624 \\
\hline 8 & +1 & +1 & +1 & 0,454 & 0,444 \\
\hline
\end{tabular}

${ }^{a}$ Cada experimento foi realizado de forma randômica e em 2 repetições. ${ }^{\mathrm{b}}(-1)$ para $4,0 \%(\mathrm{v} / \mathrm{v}) ;(+1)$ para $8,0 \%(\mathrm{v} / \mathrm{v}) .{ }^{\mathrm{c}}(-1)$ para $0,4 \%(\mathrm{~m} / \mathrm{v}) ;(+1)$ para $0,8 \%(\mathrm{~m} / \mathrm{v}) .{ }^{\mathrm{d}}(-1)$ para Fármaco $+\mathrm{HCl}+p$-DACA; $(+1)$ para Fármaco $+p$-DACA $+\mathrm{HCl}$

solução de metoclopramida na concentração de $6,21 \times 10^{-3} \mathrm{~mol} \mathrm{~L}^{-1}$. $\mathrm{O}$ volume de cada uma das soluções foi mantido fixo, sendo $10 \mu \mathrm{L}$ de cada uma das soluções a ser adicionada (metoclopramida, ácido clorídrico e $p$-DACA). Foram realizados brancos correspondentes a cada um dos experimentos.

A partir dos resultados do planejamento fatorial completo, o gráfico de Pareto (Figura 2) foi obtido para visualização dos efeitos estimados dos fatores principais. O gráfico de Pareto fornece uma representação gráfica para estes fatores e permite observar a magnitude e a importância de um determinado efeito. Neste gráfico, as barras (fatores) que graficamente ultrapassam a linha de significância exercem uma influência estatisticamente significativa sobre o resultado. Assim, pode ser claramente observado na Figura 2, que todos os fatores exerceram efeito significativo sobre a resposta. A ordem de adição foi o fator que teve maior influência, seguida pela concentração de ácido e concentração de $p$-DACA. Observa-se na Figura 2 que a ordem de adição apresentou um valor negativo, indicando que as maiores respostas foram alcançadas quando esta variável estava no nível baixo (-1), ou seja, quando a solução de fármaco foi adicionada inicialmente, seguida pelo $\mathrm{HCl}$ e, por último, $p$-DACA. Considerando estes resultados e lembrando que se trata de um fator qualitativo, esta ordem de adição foi estabelecida. Nota-se na Figura 2 que a concentração de ácido também apresentou um valor negativo, indicando que as maiores respostas foram alcançadas com a solução de $\mathrm{HCl}$ em concentrações mais baixas (-1), enquanto que a concentração do $p$-DACA apresentou valor positivo, sugerindo que

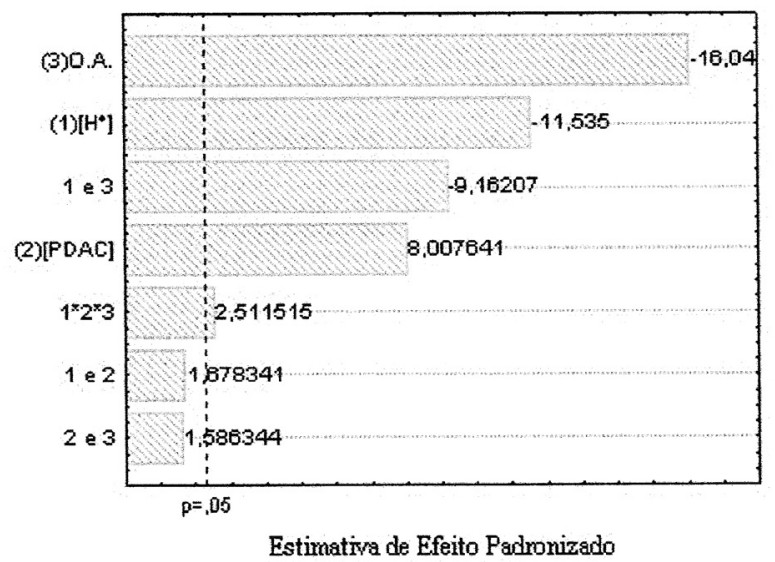

Figura 2. Gráfico de Pareto mostrando as variáveis que influenciam significativamente na reação entre metoclopramida e p-DACA, em meio ácido 
as maiores respostas foram obtidas quando esta solução estava em concentrações mais altas $(+1)$.

Com base nos resultados obtidos no planejamento fatorial completo, um planejamento composto central foi delineado visando obter as melhores condições para a reação. ${ }^{41}$ Os pontos (níveis) de um planejamento composto central estão a uma distância de $\sqrt{ } 2$ unidade (codificada) do ponto central (normalmente codificado como ponto zero); desta forma, todos os pontos estão sobre uma circunferência com raio $\sqrt{2}$. A Tabela 2 apresenta a matriz do planejamento composto central e os valores de resposta $\left(\mathrm{A}_{\mathrm{R}}\right)$ obtidos nos experimentos. $\mathrm{O}$ experimento correspondente ao ponto central foi realizado em triplicata. Uma vez que a ordem de adição havia sido estabelecida na primeira etapa, os fatores estudados no planejamento composto central foram a concentração de $\mathrm{HCl}$, variando de 1,0 a $6,0 \%(\mathrm{v} / \mathrm{v})$, e o volume da solução de $p$-DACA com concentração fixa $(0,8 \%$, $\mathrm{m} / \mathrm{v}$ ), variando de 10 a $20 \mu \mathrm{L}$.

Tabela 2. Matriz e resultados obtidos no planejamento composto central

\begin{tabular}{clll}
\hline Experimentos & {$[\mathrm{HCl}](\%, \mathrm{v} / \mathrm{v})^{\mathrm{a}}$} & $\mathrm{V}_{p \text {-DACA } 0.8 \%}(\mu \mathrm{L})^{\mathrm{a}}$ & $\mathrm{A}_{\mathrm{R}}$ \\
\hline 1 & $1,7(-1)$ & $12(-1)$ & 0,591 \\
2 & $5,3(+1)$ & $12(-1)$ & 0,657 \\
3 & $1,7(-1)$ & $18(+1)$ & 0,602 \\
4 & $5,3(+1)$ & $18(+1)$ & 0,637 \\
$5^{\mathrm{b}}$ & $3,5(0)$ & $15(0)$ & 0,673 \\
$6^{\mathrm{b}}$ & $3,5(0)$ & $15(0)$ & 0,660 \\
$7^{\mathrm{b}}$ & $3,5(0)$ & $15(0)$ & 0,654 \\
8 & $1,0(-1,41)$ & $15(0)$ & 0,633 \\
9 & $6,0(+1,41)$ & $15(0)$ & 0,643 \\
10 & $3,5(0)$ & $10(-1,41)$ & 0,623 \\
11 & $3,5(0)$ & $20(+1,41)$ & 0,653 \\
\hline
\end{tabular}

a Os valores codificados estão entre parênteses. ${ }^{\mathrm{b}}$ Média: 0,662; Variância $\left(s^{2}\right): 0,0000907$.

Os resultados obtidos a partir deste planejamento foram ajustados a um modelo matemático quadrático descrito pela Equação 1, utilizando o programa Statistica, versão 6.0.

$Y=0,66218+0,01413 x+0,0042224 x_{2}-0,016175 x_{1}^{2}-0,016165 x_{2}^{2}$ $-0,07673 x_{1} x_{2}$

A Equação 1 inclui os termos lineares $\left(x_{1}\right.$ e $\left.x_{2}\right)$ e quadráticos $\left(x_{1}^{2}\right.$ e $\left.x_{2}^{2}\right)$ bem como o produto dos pares dos efeitos lineares $\left(x_{1} x_{2}\right)$. Nesta equação, Y representa a absorbância em $580 \mathrm{~nm}$, e as variáveis $x_{1} \mathrm{e} x_{2}$ são volumes de $p$-DACA e concentração de $\mathrm{HCl}$, respectivamente.

A Figura 3 apresenta o gráfico tridimensional obtido a partir dos dados experimentais e ajustados a uma superfície de resposta juntamente com sua curva de nível desenvolvidas através do planejamento composto central cuja matriz está representada na Tabela 2. Nota-se pelo formato da superfície que a região ótima foi encontrada e também que as respostas máximas foram alcançadas com solução de $\mathrm{HCl}$ de cerca de $4,0 \%(\mathrm{v} / \mathrm{v})$ e $15 \mu \mathrm{L}$ de $p$-DACA.

\section{Curva analítica, limite de detecção e limite de quantificação}

A curva analítica foi construída utilizando soluções de metoclopramida numa faixa de concentração entre $5,65 \times 10^{-4}$ e $6,21 \times 10^{-3}$ mol L $\mathrm{L}^{-1}$. Uma relação linear $(r=0,999)$ foi obtida construindo-se uma curva de $A_{R}$ versus $\log C$, onde $A_{R}$ é a reflectância medida em 580 $\mathrm{nm}$ e $\mathrm{C}=$ [metoclopramida] $\times 10^{4} \mathrm{~mol} \mathrm{~L}^{-1}$ (o fator $10^{4}$ foi usado para ajustar a curva analítica com valores de log maiores do que zero). A equação linear obtida para a curva analítica foi: $A_{R}=-0,15048+$
0,4354 x C. O limite de detecção $\left(3 \mathrm{xDP}_{\mathrm{h}} / \mathrm{a}\right)$ e o limite de quantificação $\left(10 \times \mathrm{XP}_{\mathrm{b}} / \mathrm{a}\right)$ obtidos foram $1,27 \times 10^{-4}$ e $4,19 \times 10^{-4} \mathrm{~mol} \mathrm{~L}^{-1}$, respectivamente, sendo a $=$ coeficiente angular da curva analítica $\mathrm{e}$ $\mathrm{DP}_{\mathrm{b}}=$ desvio padrão do branco para 10 medições. ${ }^{42}$

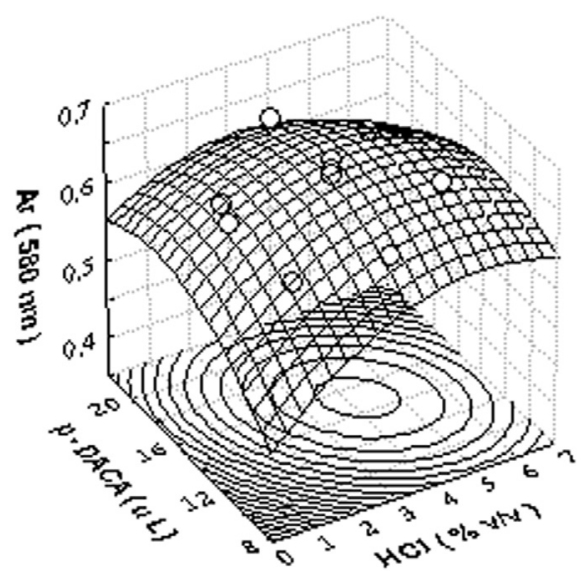

Figura 3. Superfície de resposta obtida no planejamento composto central para valores de $A_{R}$ em função da concentração de $\mathrm{HCl}$ e volume de $\mathrm{p}$-DACA $(0,8 \%, \mathrm{~m} / \mathrm{v})$. [Metoclopramida] $=6,21 \times 10^{-3} \mathrm{~mol} \mathrm{~L}^{-1}$ e volume fixado em $10 \mu \mathrm{L}$

\section{Estudo da estabilidade óptica}

Os resultados obtidos no estudo de estabilidade do produto da reação entre metoclopramida e $p$-DACA em meio ácido demonstraram que não houve diferença significativa entre os valores de $A_{R}$ obtidos no período de tempo estudado (60 $\mathrm{min})$.

\section{Estudo dos interferentes}

O efeito de cada excipiente (estearato de magnésio, amido, lactose, croscarmelose sódica, polivinilpirrolidona, manitol, metabissulfito de sódio e talco) foi considerado como interferência quando o sinal de $A_{R}$ apresentou um erro maior ou igual a 5\% na determinação do fármaco. Nenhuma interferência foi constatada a partir destes excipientes, sob as c ondições estudadas.

\section{Adição de padrão}

Com o objetivo de avaliar as possíveis interferências provenientes da matriz foi realizada a adição de padrão utilizando 3 amostras de diferentes marcas comerciais, sendo duas delas comprimidos e uma injetável. Os resultados obtidos estão apresentados na Tabela 3. Para todas as amostras os valores de recuperação variaram de 95,1 a 104,5\%, indicando boa exatidão do método e ausência de efeito significativo de matriz.

\section{Aplicação analítica}

O método proposto foi aplicado na determinação de metoclopramida em formulações farmacêuticas disponíveis comercialmente e os resultados comparados com os obtidos por métodos para a determinação de metoclopramida em comprimidos e em injetáveis descritos, respectivamente, na Farmacopeia Brasileira ${ }^{21}$ e na Farmacopeia Britânica. ${ }^{22}$ As análises foram realizadas em triplicata e os resultados estão apresentados na Tabela 4.

Para todas as formulações farmacêuticas analisadas os resultados obtidos pelo método proposto e pelos métodos oficiais das Farmacopeias Brasileira e Britânica foram comparados estatisticamente 
Tabela 3. Resultados de recuperação de metoclopramida adicionada em formulações farmacêuticas

\begin{tabular}{lcccc}
\hline Amostras & $\begin{array}{c}\text { Valor } \\
\text { adicionado } \\
\left.(\mathrm{mol} \mathrm{L})^{-1}\right)\end{array}$ & $\begin{array}{c}\text { Valor } \\
\text { esperado } \\
\left(\mathrm{mol} \mathrm{L} \mathrm{L}^{-1}\right)\end{array}$ & $\begin{array}{c}\text { Valor } \\
\text { encontrado } \\
\left(\mathrm{mol} \mathrm{L} \mathrm{L}^{-1}\right.\end{array}$ & $\begin{array}{c}\text { Recuperação } \\
(\%)\end{array}$ \\
\hline A & $8,47 \times 10^{-4}$ & $2,54 \times 10^{-3}$ & $2,47 \times 0^{-3}$ & 97,2 \\
& $1,69 \times 10^{-3}$ & $3,39 \times 10^{-3}$ & $3,29 \times 10^{-3}$ & 97,0 \\
& $2,54 \times 10^{-3}$ & $4,23 \times 10^{-3}$ & $4,38 \times 10^{-3}$ & 103,5 \\
& $3,39 \times 10^{-3}$ & $5,08 \times 10^{-3}$ & $5,31 \times 10^{-3}$ & 104,5 \\
B & $8,47 \times 10^{-4}$ & $2,54 \times 10^{-3}$ & $2,45 \times 10^{-3}$ & 96,4 \\
& $1,69 \times 10^{-3}$ & $3,39 \times 10^{-3}$ & $3,33 \times 10^{-3}$ & 98,2 \\
& $2,54 \times 10^{-3}$ & $4,23 \times 10^{-3}$ & $4,17 \times 10^{-3}$ & 98,6 \\
& $3,39 \times 10^{-3}$ & $5,08 \times 10^{-3}$ & $5,07 \times 10^{-3}$ & 99,8 \\
& $8,47 \times 10^{-4}$ & $2,54 \times 10^{-3}$ & $2,43 \times 10^{-3}$ & 95,7 \\
& $1,69 \times 10^{-3}$ & $3,39 \times 10^{-3}$ & $3,27 \times 10^{-3}$ & 96,5 \\
& $2,54 \times 10^{-3}$ & $4,23 \times 10^{-3}$ & $4,31 \times 10^{-3}$ & 101,9 \\
& $3,39 \times 10^{-3}$ & $5,08 \times 10^{-3}$ & $4,83 \times 10^{-3}$ & 95,1 \\
\hline
\end{tabular}

Tabela 4. Determinação de metoclopramida em formulações farmacêuticas comerciais pelo método proposto e pelos métodos oficiais

\begin{tabular}{ccccc}
\hline Amostras & $\begin{array}{c}\text { Método } \\
\text { proposto }^{\mathrm{c}}\end{array}$ & $\mathrm{t}(2,78)^{\mathrm{d}}$ & $\mathrm{F}(19,00)^{\mathrm{d}}$ & $\begin{array}{c}\text { Método } \\
\text { oficial }^{21,22}\end{array}$ \\
\hline $\mathrm{A}^{\mathrm{a}}$ & $10,1 \pm 0,3$ & 0,19 & 0,11 & $10,1 \pm 0,1$ \\
$\mathrm{~B}^{\text {a }}$ & $10,1 \pm 0,4$ & 0,12 & 0,63 & $10,1 \pm 0,1$ \\
$\mathrm{C}^{\text {a }}$ & $9,9 \pm 0,1$ & 2,74 & 1,00 & $9,7 \pm 0,1$ \\
D $^{\mathrm{b}}$ & $10,0 \pm 0,3$ & 0,82 & 0,11 & $9,8 \pm 0,1$ \\
E $^{\mathrm{b}}$ & $10,1 \pm 0,3$ & 0,75 & 0,11 & $10,2 \pm 0,1$ \\
\hline
\end{tabular}

aValor nominal: $10 \mathrm{mg}$ de metoclopramida/comprimido; ${ }^{\mathrm{b}}$ valor nominal: 10 $\mathrm{mg}$ de metoclopramida/ $2 \mathrm{~mL}$ solução; ' ${ }^{2}$ édia \pm desvio padrão $(\mathrm{mg}), \mathrm{n}=3$; ${ }^{\mathrm{d}}$ valores tabelados de t e F no nível de confiança de $95 \%$.

aplicando-se o teste t e o teste F, em um nível de confiança de $95 \%$. Em todos os casos, os valores de t e de $\mathrm{F}$ calculados não excederam os valores tabelados indicando que não existe diferença significante com respeito à exatidão e precisão entre o método proposto e os métodos oficiais, em um nível de confiança de 95\% (Tabela 4).

\section{CONCLUSÕES}

Este estudo demonstrou a viabilidade de se empregar a espectroscopia de reflectância difusa combinada com spot test na determinação de metoclopramida em formulações farmacêuticas. O método proposto apresenta grande vantagem sobre os métodos oficiais por espectrofotometria no UV descritos nas Farmacopeias Brasileira e Britânica, pois não tem interferência de excipientes e, portanto, não necessita de extrações prévias com clorofórmio, solvente nocivo ao operador e ao ambiente. Adicionalmente, também apresenta vantagens sobre outros métodos descritos na literatura como simplicidade, rapidez, portabilidade e, principalmente, o baixo consumo de solventes e reagentes. Trata-se, portanto, de um método mais atrativo e ambientalmente mais amigável.

\section{AGRADECIMENTOS}

Ao CNPq e à FAPESP pelo suporte financeiro deste trabalho.

\section{REFERÊNCIAS}

1. Martindale. The Extra Pharmacopoeia, $29^{\text {th }}$ ed., The Pharmaceutical Press: London, 1989.
2. Herrero, M. R.; Romero, A. M.; Calatayud, J. M.; Talanta 1998, 47, 223.

3. Omran, A. A.; Chem. Pharm. Bull. 2005, 53, 1498.

4. Shah, J.; Jan, M. R.; Khan, M. A.; Amin, S.; J. Anal. Chem. 2005, 60, 633.

5. Abdel-Gawad, F. M.; El-Guindi; N. M.; Anal. Lett. 1995, 28, 1437.

6. El-Gendy, A. E.; Spectrosc. Lett. 1992, 25, 1297.

7. Sastry, C. S. P.; Kumari, P. L.; Rao, B. G.; Chem. Anal. 1985, 30, 461.

8. Revanasiddappa, H. D.; Manju, B. J.; J. Pharm. Biomed. Anal. 2001, 25, 631.

9. Moussa, B. A.; J. Pharm. Biomed. Anal. 2000, 23, 1045.

10. Amin, A. S.; Ragab, G. H.; Anal. Sci. 2003, 19, 747.

11. Buna, M.; Aaron, J. J.; Prognon, P., Mahuzier, G., Analyst 1996, 121, 1551.

12. Venkateshwaran, T. G.; Kimng, D. T.; Stewart, J. T.; J. Liq. Chromatogr. 1995, 18, 117

13. Foda, N. H.; Anal. Lett. 1994, 27, 549.

14. El-Sayed, Y. M.; Khidr, S. H.; Naizy, E. M.; Anal. Lett. 1994, 27, 55.

15. Díaz, C.; Vidal, J. C.; Galban, J.; Lanaja J.; J. Electroanal. Chem. 1989, 258, 295.

16. Badawy, S. S.; Shoukry A. F.; Issa, Y. M.; Analyst 1986, 111, 1363.

17. Badwan, A. A.; Jawan, O. A.; Owais, L.; Int. J. Pharm. 1986, $28,41$.

18. Mostafa, G. A. E.; J. Pharm. Biomed. Anal. 2003, 31, 515.

19. Hanna, G. M.; Lau-Cam, C. A.; Drug Dev. Ind. Pharm. 1991, 17, 975.

20. Norouzi, P.; Ganjali, M. R.; Matloobi, P.; Electrochem. Commun. 2005, $7,333$.

21. Farmacopéia Brasileira, $4^{\mathrm{a}}$ ed., Atheneu: São Paulo, 2003.

22. British Pharmacopoeia; British Pharmacopeia Commission, H.M.S.O: London, 2001, vol. II.

23. The United States Pharmacopoeia, $24^{\text {th }}$ ed., The United States Pharmacopoeial Convention: Rockville, 2000.

24. Anastas, P. T.; Crit. Rev. Anal. Chem. 1999, 29, 167.

25. Tubino, M.; Rossi, A. V.; Magalhães, M. E. A.; Anal. Lett. 1997, 30, 271.

26. Matias, F. A. A.; Vila, M. M. D. C.; Tubino, M.; J. Braz. Chem. Soc. 2004, 15, 327.

27. Gotardo, M. A.; Gigante, A. C.; Pezza, L.; Pezza, H. R.; Talanta 2004, 64, 361.

28. Ribeiro, P. R. S.; Pezza, L.; Pezza, H. R.; J. Braz. Chem. Soc. 2006, 17, 679.

29. Ghauch, A.; Turnar, C.; Fachinger, C.; Rima, J.; Charef, A.; Suptil, J.; Mantin-Bouyer, M.; Chemosphere 2000, 40, 1327.

30. Matias, F. A. A.; Vila, M. M. D. C.; Tubino, M.; Sens. Actuators B 2003, 88,60 .

31. Arena, M. P.; Porter, M. D.; Fritz, J. S.; Anal. Chem. 2002, 74, 185.

32. Gazda, D. B.; Fritz, J. S.; Porter, M. D.; Anal. Chem. 2004, 76, 4881.

33. Gotardo, M. A.; Tognolli, J. O.; Pezza, H. R.; Pezza, L.; Spectrochim. Acta, Part A 2008, 69, 1103.

34. Weinert, P. L.; Pezza, L.; Pezza, H. R.; J. Braz. Chem. Soc. 2007, 18, 846.

35. Tubino, M.; Souza, R. L.; Talanta 2006, 68, 776.

36. Thompson, M.; Ellison, S. L. R.; Wood, R.; Pure Appl. Chem. 2002, 74, 835.

37. Saeed, A.; Haque, S.; Qureshi, S. Z.; Talanta 1993, 40, 1867.

38. El Sherif, Z. A.; Walash, M. I.; El-Tarras, M. F.; Osman, A. O.; Anal. Lett. 1997, 30, 1881.

39. Zawilla, N. H.; Mohammad, M. A. A.; El Kousy, N. M.; El-Moghazy, A. S. M.; J. Pharm. Biomed. Anal. 2002, 27, 243.

40. Wendlant, W. W.; Hecht, H. G.; Reflectance spectroscopy, Interscience Publishers: New York, 1966.

41. Barros Neto, B.; Scarminio, I. S.; Bruns, R. E.; Como fazer experimentos: Pesquisa e desenvolvimento na ciência e indústria, $2^{\mathrm{a}}$ ed., Ed. da Unicamp: Campinas, 2003.

42. Long, G. L.; Winefordner, J. D.; Anal. Chem. 1983, 55, 712. 